\title{
WORKSHOP SUMMARY: EXPERIMENT
}

\author{
KENNETH HICKS * \\ Department of Physics and Astonomy \\ Athens, $\mathrm{OH}$ 45701, USA \\ E-mail: hicks@ohio.edu
}

\begin{abstract}
A summary of experimental results from the Pentaquark 2004 Workshop held at the SPring-8 facility in Japan is given. New results from the LEPS collaboration are highlighted, and older results are reviewed. Non-observations are also discussed in light of theoretical estimates of possible $\Theta^{+}$production mechanisms. The problem of the narrow width and the parity of the $\Theta^{+}$are explored and point to future experimental work that is needed.
\end{abstract}

\section{Introduction}

This was an exciting workshop with many new results in the rapidly changing field of exotic baryons. Both theoretical and experimental advances have been made, with new ideas by the theorists to explain how a very narrow resonance can be constructed. On the other hand, the experimental results are mixed, with some new positive evidence and some new null measurements, and little hope to clarify the questions of width and parity of the $\Theta^{+}$ within the next few years. Clearly, the existence of the pentaquark is an experimental issue, and it must be resolved before the physics community can take seriously the theoretical explanations. Here, I will focus on the experimental results and leave the theoretical summary to Carlson ${ }^{1}$.

With both positive and null measurements of several possible pentaquark resonances, there are strong statements being made on both sides of the argument. In fact, some might even say that it has become an emotionally-charged issue in hadronic physics. It is important to realize that it takes time to do good experiments, and that nature sometimes has surprises in store for us. For this reason, caution and patience are advised while we wait for progress. If we let science take its course, then in the end the truth will emerge.

*Supported in part by RCNP (Japan) and the NSF/DOE (USA). 
Since there is uncertainty in the existence of the pentaquark, the best we can do is ask whether there is good reason to be optimistic or pessimistic for the future of the pentaquark. I will return to this question periodically.

\section{Reasons to be Optimistic}

Over ten experiments have published evidence for the $\Theta^{+}$pentaquark $2,3,4,5,6,7,8,9,10,11,12$. While the number of measurements may be impressive, one must keep in mind that most of these are in the range of 4-6 standard deviations above a background that is difficult to quantify. In fact, these statistical estimates assume a smooth background shape, and hence the statistical significance may be overestimated. Still, it seems unlikely that all of the results can be explained as a statistical fluctuation of the background, since so many different reactions (with different backgrounds) have been used in the analysis. Of course, such hand-waving arguments are not proof that the $\Theta^{+}$exists, but the variety of reactions and the quality of the experiments does provide some encouragement that the $\Theta^{+}$exists.

The HERMES and ZEUS experiments are well-known and respected (as are other groups that have published positive evidence). However, these experiments have been criticized ${ }^{13}$ because they cannot determine the strangeness in the $K^{0} p$ final state. In this workshop, the HERMES collaboration ${ }^{14}$ showed that the $K^{0} p$ peak in their results is not consistent with an interpretation as a $\Sigma^{*+}$ resonance. They also showed that the peak to background ratio in their data can be enhanced by applying cuts that remove the known $K^{*}$ and $\Lambda^{*}$ resonances. This strengthens their case that the peak is real, although more statistics are needed, which will occur in the next year. This is some reason for optimism.

An intriguing result was presented by the GRAAL collaboration ${ }^{15}$ which did not search for the $\Theta^{+}$but instead have evidence for a narrow $N^{*}$ resonance near $1680 \mathrm{MeV}$. In their measurement, $\eta$-photoproduction on deuterium, they separate events that occurred on the neutron or the proton. In a theoretical prediction by Polyakov and Rathke ${ }^{16}$, transitions from octet baryons to antidecuplet baryons are suppressed on the proton (due to an isospin factor) but allowed on the neutron. In fact, this is what the GRAAL collaobration see, albeit with limited statistics. If this claim can be confirmed by other experiments, then this narrow $N^{*}$ resonance fits better into the $\overline{10}$ symmetry group that includes the $\Theta^{+}$as well. But until the GRAAL result is confirmed, it is wise to resist the urge for optimism.

Perhaps the best evidence so far for the $\Theta^{+}$was shown at this work- 
shop by the LEPS collaboration ${ }^{17}$. This analysis of the $\gamma d \rightarrow K^{+} K^{-} X$, where $X$ is restricted to have the mass of deuterium, is an improvement over analysis shown at the MESON04 conference ${ }^{13}$. Additional cuts to remove coherent production and an energy-dependent $\phi$-meson exclusion were shown to enhance the $\Lambda(1520)$ peak in the $K^{+}$missing mass spectrum (corrected for Fermi motion). These same cuts also enhance the peak in the $K^{-}$missing mass, where the $\Theta^{+}$might be expected. A new development is the use of event mixing to get the shape of the background. Event mixing uses a $K^{+}$from one event and a $K^{-}$from a different event. The missing mass of the mixed events is still required to be at the deuterium mass, which ensures energy conservation. The advantage of using event mixing is: (1) the statistics can be increased because of the number of combinations, (2) the real angle and momentum distribution is used for the kaons, which is better than a phase-space Monte Carlo that is sometimes used to determine the detector acceptance, (3) correlations between the $K^{+}$and $K^{-}$are removed, so that the generated background goes smoothly under real peaks (which by definition have correlated $K^{+} K^{-}$pairs). The event mixing was shown ${ }^{17}$ to work for the background under the $\Lambda(1520)$ and using the same procedure, the $\Theta^{+}$peak also comes up clearly above the mixed-event background. This quantitative analysis of the background shape gives more credence to the $\Theta^{+}$peak seen in the new LEPS deuterium data.

Finally, the best reason for optimism is that there are several new results on the horizon that have the potential to convincingly settle the question of whether the $\Theta^{+}$exists or not. The CLAS collaboration has taken new data on both a deuterium target ${ }^{18}$ and a proton target ${ }^{19}$ with about 10 times the statistics of earlier data, and expect to have results on several different $\Theta^{+}$search topologies by early 2005 . The COSY-TOF collaboration ${ }^{20}$ will upgrade their detector and will take more data in 2005, thus increasing their statistics by (perhaps) a factor of five. As already mentioned, the HERMES collaboration will double their statistics on a deuterium target soon, which can substantially help their $\Theta^{+}$search. With these new results on the horizon, 2005 will be an exciting year.

\section{Reasons to be Pessimistic}

There are a number of experiments that give null results in a search for the $\Theta^{+}$pentaquark. At the time of this conference, only 2 were published ${ }^{21,22}$ and a number had been presented at an earlier conference ${ }^{23}$. At this 
meeting, there were presentations of pentaquark searches by the BaBar ${ }^{24}$, Belle ${ }^{25}$ and Fermilab E690 ${ }^{26}$ collaborations. Naively, one would expect to see the $\Theta^{+}$(and perhaps the $\Xi^{--}$) in these experiments, and the null results imply that either: (1) the pentaquark does not exist, or (2) the production mechanism of pentaquarks differs from that of 3-quark baryon resonances. In any case, these results are good reason for one to be pessimistic (or at the very least very cautious) about the existence of pentaquarks.

The BaBar results ${ }^{24}$ have high statistics and reasonable signals for the established $N^{*}$ and $Y^{*}$ resonances. Here, a baryon-antibaryon pair is created from $e^{+} e^{-}$collisions at $\sqrt{s}=10.58 \mathrm{GeV}$. The $\Lambda(1520)$ resonance is seen clearly in the $p K^{-}$invariant mass, but no structure is seen in the $p K^{0}$ mass spectrum in the region of the $\Theta^{+}$mass. Based on systematics of baryon production rates as a function of baryon mass, one can estimate the number of $\Theta^{+}$baryons that should have been produced. However, this assumes that pentaquark production (involving 5 quarks and 5 antiquarks) follows the same systematics as 3-quark baryons. Due to the uncertainty in the production mechanism, theoretical calculations are needed to understand the true significance of these null results.

The Belle experiment ${ }^{25}$ took a different approach. They used secondary scattering of mesons (from $e^{+} e^{-}$collisions) in their silicon vertex detector to produce known $Y^{*}$ resonances. If the $\Theta^{+}$exists, it could be produced with a $K^{+}$beam of the right energy. Unfortunately, the hadrons incident on the silicon target have unknown identity and unknown energy. Only a small fraction of these data could result in production of the $\Theta^{+}$and detected by its decay into the $p K^{0}$ channel. With the high resolution of Belle, even a small signal (with a narrow width) might be visible, but none was seen. Again, we need better calculations of the expected number of counts (based on Belle's spectrum of hadrons incident on silicon) before we can interpret their null result.

The E690 experiment ${ }^{26}$ uses protons of about $800 \mathrm{GeV}$ in peripheral collisions with target protons. By putting cuts on the missing transverse momentum and the longitudinal energy, exclusive reactions can be measured. In the $p K^{-}$mass spectrum, about $5000 \Lambda(1520)$ events are seen, but no structure is seen in the $p K^{0}$ mass spectrum. Because of the exclusive reaction, the strangeness of the $p K^{0}$ system is known and so this spectrum is not contaminated with $\Sigma^{*}$ resonances, such as the $\Sigma(1660)$. Hence, this is a significant null result, and suggests one should be pessimistic about the $\Theta^{+}$existence.

One interesting development of this workshop was a calculation pre- 
sented by Titov ${ }^{27}$, using quark constituent counting rules to estimate the ratio of $\Theta^{+}$to $\Lambda(1520)$ production in fragmentation reactions. Fragmentation functions are well established ${ }^{28}$ and have been used for years to describe the distribution of hadrons from high-energy collisions, based on the number of constituent partons in the projectile and target. Using this model, Titov shows that production of the $\Theta^{+}$is suppressed relative to the $\Lambda(1520)$ resonance by about 3 orders of magnitude for experiments such as E690 and BaBar. Of course, the simple model used for this estimate may not be a good approximation for all kinematics, but it is consistent with the null experimental results at high energies. The optimist would argue that we could have expected null results from fragmentation reactions in high-energy experiments.

It is easy to see that there is reason for pessimism, but the evidence is not entirely convincing. It is difficult to know how many $\Theta^{+}$events should have been seen in the high-energy experiments with null results. In fact, there is even a reasonable explanation from Titov, using fragmentation functions, for these null results. The case for "killing" the $\Theta^{+}$has not been made.

\section{The Problem of the Width}

Perhaps the most disturbing fact of the $\Theta^{+}$evidence is that its width appears to be very narrow. Direct evidence ${ }^{3,8,9}$ limits the width to be less than about $10 \mathrm{MeV}$. Indirect evidence, based on analysis of $\mathrm{KN}$ scattering data $29,30,31,32,33$, estimates the width at a few MeV or less. Such a narrow width for a resonance $100 \mathrm{MeV}$ above its strong decay threshold would be unprecedented.

Coupled with the narrow width problem is the question of parity. The spin of the lowest-lying $\Theta^{+}$is expected to be $J=1 / 2$ with either negative (S-wave) or positive (P-wave) parity. A narrow width from an $\mathrm{S}$-wave resonance makes no sense ${ }^{34}$ whereas a $\mathrm{P}$-wave would allow a centrifugal barrier making a narrow width at least possible ${ }^{34,35}$. It seems logical that if the $\Theta^{+}$width is narrow, its parity must be positive. This idea was beautifully presented by Hosaka ${ }^{36}$.

What do lattice QCD calculations say about the parity? Several lattice results were presented at this conference, and except for one result ${ }^{37}$, only the negative parity projection gives a result consistent with the $\Theta^{+38,39}$. So we have an apparent contradiction between the parity deduced from quark models (above) and the parity deduced from (most) lattice calculations. 
One obvious resolution to this dilemma is to conclude that the $\Theta^{+}$does not exist. However, we must realize that the lattice calculations for exotic baryon resonances should be regarded as exploratory ${ }^{38}$. Extrapolating to the chiral limit from the heavy quarks used in lattice calculations must be done properly ${ }^{37,39}$ and furthermore, all lattice calculations are done in the quenched approximation. Hence we should be cautious about parity statements based on current lattice results.

Even if the $\Theta^{+}$exists with positive parity, a width as narrow as $1 \mathrm{MeV}$ is theoretically difficult to understand ${ }^{40}$. However, several new theoretical ideas were presented showing that such a narrow width is consistent with theory. Using a two-state model, Lipkin showed ${ }^{41}$ that the mass eigenstates of two pentaquarks (e.g., mixtures of the Jaffe-Wilczek model and the diquark-triquark model) can mix, resulting in one coupling strongly to KN decay (with a wide width) and one decoupling (with a narrow width). Another approach, this time with mixing between the octet and the $\overline{10}$, shown by Praszalowicz ${ }^{40}$, can suppress the width by a correction factor that depends on the value of the pion-nucleon $\Sigma$ term. From a completely different angle, using the QCD string model, Suganuma et al. showed ${ }^{42}$ that the pentaquark does not just "fall apart" as predicted by the quark model, but must overcome a sizeable potential barrier to decay into a $\mathrm{KN}$ final state. This results in a very narrow width for the $\Theta^{+}$in their model. In all, it is interesting that a narrow width of $1 \mathrm{MeV}$ can be accomodated in the quark model, the chiral soliton model and the QCD string model.

Clearly, experimental information is needed before one can test the various ideas about the $\Theta^{+}$width. Proposals at KEK ${ }^{43}$ and Jefferson Lab ${ }^{44}$ for high resolution spectrometer experiments are being considered. Other facilities already mentioned (COSY-TOF, HERMES, ZEUS) will gather more statistics, which should enable a better determination of the $\Theta^{+}$width. In addition to width measurements, we need to know the $\Theta^{+}$parity. This will likely be done at COSY-TOF ${ }^{20}$ using polarized target and polarized beam, which has a clear theoretical interpretation as shown by Hanhart ${ }^{45}$. If the $\Theta^{+}$exists, then we have the experimental tools to learn about its width and parity.

\section{Summary}

This workshop was filled with exciting new developments, both experimental and theoretical. The LEPS collaboration showed new data, this time using a deuterium target, which appears to confirm the existence of the $\Theta^{+}$ 
although the results are still preliminary. A possible narrow $N^{*}$ state was seen in $\eta$ photoproduction from the neutron at GRAAL, but not from the proton, in agreement with theoretical predictions. While no new data were shown for the $\Theta^{+}$width or parity, several theoretical models showed that a narrow width for the $\Theta^{+}$is not unreasonable.

The null results from high-energy experiments are worrisome, but theoretical estimates from fragmentation functions suggest that $\Theta^{+}$production is very suppressed relative to baryon resonances like the $\Lambda(1520)$. If so, then this is not negative evidence for the $\Theta^{+}$but just non-observation. Of course, the proof of $\Theta^{+}$existence must be convincing in the medium energy experiments, with high-statistics data, before one can believe it is suppressed in high-energy data.

So should we be optimistic or pessimistic about the existence of pentaquarks? At the present time, there is no clear choice. However, new data will be available soon that will clarify the situation. If the $\Theta^{+}$exists, then we have a rich new spectroscopy to explore.

\section{Acknowledgments}

I am grateful for the support I received from RCNP (Osaka University) and Kyoto University during my sabbatical in Japan. It has been a great pleasure to work closely with Takashi Nakano and Atsushi Hosaka, along with the members of their groups, during my stay. I congratulate Hosakasan for the excellent organization of the Pentaquark 2004 workshop.

\section{References}

1. C. Carlson, these proceedings.

2. T. Nakano et al., (LEPS), Phys. Rev. Lett. 91, 012002 (2003).

3. V.V. Barmin et al., (DIANA), Phys. Atom. Nuclei 66, 1715 (2003).

4. S. Stepanyan et al., (CLAS), Phys. Rev. Lett. 91, 25001 (2003).

5. V. Kubarovsky et al., (CLAS), Phys. Rev. Lett. 92, 032001 (2004).

6. J. Barth et al., (SAPHIR), Phys. Lett. B572, 127 (2003).

7. A.E. Asratyan, A.G. Dolgolkenko and M.A. Kubantsev, Phys. Atom. Nucl. (2004); hep-ex/0309042.

8. A. Airapetian et al., (HERMES), Phys. Lett. B585 (2004) 213; hepex/0312044.

9. The ZEUS collaboration, Phys. Lett. B591 (2004) 7-22; hep-ex/0403051.

10. C. Alt et al., (NA49), Phys. Rev. Lett. 92, 042003 (2004); hep-ex/0310014.

11. M. Abdel-Barv, et al., (COSY-TOF), Phys. Lett. B595, 127 (2004); nuclex/0403011.

12. A. Aleev et al., (SVD), submitted to Yad. Fiz.; hep-ex/0401024. 
13. K. Hicks, hep-ph/0408001.

14. W. Lorenzon (HERMES), these proceedings.

15. C. Schaerf (GRAAL), these proceedings.

16. M.V. Polyakov and A. Rathke, hep-ph/0303138.

17. T. Nakano et al., these proceedings.

18. D. Tedeschi (CLAS), these proceedings.

19. R. DeVita (CLAS), these proceedings.

20. W. Eyrich (COSY-TOF), these proceedings.

21. J.Z. Bai et al., (BES), hep-ex/0402012.

22. K.T. Knöpffe et al., (HERA-B), hep-ex/0403020.

23. Quarks and Nuclear Physics, Indiana University, 2004; website www.qnp2004.org.

24. V. Halyo (BaBar), these proceedings.

25. R. Mizuk (Belle), these proceedings.

26. E. Gottschalk (E690), these proceedings.

27. A.I. Titov, A. Hosaka, S. Date, Y. Ohashi, these proceedings and Phys. Rev. C 71 (2004).

28. F.E. Close, An Introduction to Quarks and Partons, Academic Press, London, 1979.

29. S. Nussinov, hep-ph/0307357.

30. R.N. Cahn and G.H. Trilling, Phys. Rev. D69, 011501 (2004).

31. R.A. Arndt, I.I. Strakovsky and R.L. Workman, Phys. Rev. C68, 042201(R) (2003).

32. A. Sibirtsev, J. Haidenbauer, S. Krewald and Ulf-G. Meissner, hep$\mathrm{ph} / 0405099$.

33. W.R. Gibbs, nucl-th/0405024.

34. R. Jaffe and F. Wilczek, Phys. Rev. Lett. 91:232003 (2003).

35. M. Karliner and H.J. Lipkin, Phys. Lett. B575 (2003) 249; hep-ph/0307243.

36. S.-I. Nam, A. Hosaka, and H.-C. Kim, these proceedings.

37. T.-W. Chiu and T.-H. Hsieh, these proceedings.

38. S. Sasaki, these proceedings.

39. F.X. Lee, these proceedings.

40. M. Praszalowicz, these proceedings.

41. H. Lipkin, these proceedings; see also hep-ph/0401072.

42. H. Suganuma, H. Ichie, F. Okiharu, T. Takahashi, these proceedings; hep$\mathrm{ph} / 0412271$.

43. K. Imai et al., KEK experiment E559.

44. B. Wojtsekhowski and G. Cates, proposal P05-009.

45. C. Hanhart, J. Haidenbauer, K. Nakayama, U.-G. Meissner, these proceedings; hep-ph/0407107. 